\title{
Obesity and Asthma: Is Diet a Therapeutic Mean?
}

George Antonogeorgos and Demosthenes B. Panagiotakos*

Department of Nutrition and Dietetics, Harokopio University, Athens, Greece

Asthma and obesity have emerged as significant epidemics in the later decades, especially in the western world. The concomitant increasing trend in the prevalences of these two chronic conditions and the underlying inflammatory mechanisms that are implicated in their pathophysiology, has led researchers in the investigation for a possible causal link between them [1-2]. Several biochemical mediators associated with obesity, such as hormones (leptin) and cytokines (interleukin- 6 and tumor necrosis factor-a) have been found elevated in asthmatic patients [3-4]. These mediators could potentially affect the airway smooth muscles and subsequently reduce the air flow, causing the dramatic arise of asthmatic symptoms such as cough, wheezing, shortness of breath and if untreated, respiratory distress and respiratory shock. Other possible pathway connecting asthma with obesity is through the respiratory mechanics and how they are affected especially by abdominal obesity [5]. Lastly, obesity had been found to be an independent predisposal factor for atopy, suggesting another link with asthma [6].

The low-grade inflammation observed in obesity and asthma could be associated with changes in common environmental factors such as diet. There are several proposed hypotheses relating similar factors in both diseases like decreases in antioxidants (vitamin C, vitamin E, carotenoids, Selenium, flavonoids and antioxidant-rich fruits), Poly-Unsaturated Fatty Acids (PUFAs) and Vitamin D blood concentrations [7-10]. However, most of the evidence in the literature is from observational and mainly cross-sectional and case-control studies, which are prone to several biases like measurement, selection and reverse causality bias.

Very small number of intervention studies, relatively to the large number of observational ones, has assessed the potentially therapeutic effect of the supplementation of antioxidants, PUFAs and Vitamin D with asthma. Vitamin C supplementations have been extensively studied in various doses but no sufficient evidence is found in order to have a place as an adjunct treatment against asthma [11]. PUFAs have been used as supplementation in randomized clinical trials but with also conflicting results [12]. Data for the potential protective role of Vitamin D are coming from observational studies solely, because the proposed therapeutic dose is too high (>2000 IU/day) and above the currently recommended dose ( $200 \mathrm{IU} /$ day from infancy to 50 years) [13]. Regarding obesity, consumption of dried fruits, a source rich in antioxidants such as vitamin $\mathrm{C}$ and $\mathrm{E}$, has been associated with reduced obesity in adults, but only through the mediation of improved diet quality [14].

However, human nutrition is a composite and should not be considered just as a daily nutrient replacement. The approach of assessing single nutrients or food items, instead of assessing dietary patterns could introduce some bias since we are not consuming isolated nutrients, but eat meals consisting of a variety of foods with complex combinations of them. A dietary pattern, the Mediterranean diet, has emerged lately in the literature as a protective factor against childhood and adult asthma, in addition to the established protective risk reduction in the cardiovascular diseases and obesity [15]. Mediterranean diet is characterized mainly by high consumption of fruits, vegetables, grains, legumes, low consumption of red meat and higher consumption of fish and white meat [16]. Mediterranean diet is also something more than just adequate intake of macro- or micronutrients, but it is also associated with a healthier lifestyle profile, expanding from increased physical activity to overall better nutritional knowledge. Adopting Mediterranean diet as a way-of-living could help restraining the inflammatory cascade of asthma, obesity and all the related complications better than it could be achieved by simply the replacement of the deficient nutrients.

Thus, the study of dietary patterns instead of isolated nutrients could offer better insights about the common role of diet in the etiopathology of obesity and asthma, providing us with new possible therapeutic means. A growing amount of evidence expands the well-known protective role of a specific dietary pattern, the Mediterranean diet, from the traditional field of obesity and cardiovascular diseases, to the new area of asthma. More intervention studies are needed in order to elucidate the beneficial effect of the Mediterranean diet in reducing the burden of these modern epidemics.

\section{References}

1. Hasler G, Gergen PJ, Ajdacic V, Gamma A, Eich D, et al. (2006) Asthma and body weight change: a 20 -year prospective community study of young adults. Int J Obes (Lond) 30: 1111-1118.

2. To T, Vydykhan TN, Dell S, Tassoudji M, Harris JK (2004) Is obesity associated with asthma in young children? J Pediatr 144: 162-168.

3. Sood A (2005) Does obesity weigh heavily on the health of the human airway? J Allergy Clin Immunol 115: 921-924.

4. Shore SA, Fredberg JJ (2005) Obesity, smooth muscle, and airway hyperresponsiveness. J Allergy Clin Immunol 115: 925-927.

5. Wannamethee SG, Shaper AG, Whincup PH (2005) Body fat distribution, body composition, and respiratory function in elderly men. Am J Clin Nutr 82: 9961003.

6. Silverberg JI, Silverberg NB, Lee-Wong M (2012) Association between atopic dermatitis and obesity in adulthood. Br J Dermatol 166: 498-504.

7. Awad AB, Alappat L, Valerio M (2012) Vitamin d and metabolic syndrome risk factors: evidence and mechanisms. Crit Rev Food Sci Nutr 52: 103-112.

8. Laerum BN, Wentzel-Larsen T, Gulsvik A, Omenaas E, Gíslason T, et al. (2007) Relationship of fish and cod oil intake with adult asthma. Clin Exp Allergy 37: 1616-1623.

9. Al-Musharaf S, Al-Othman A, Al-Daghri NM, Krishnaswamy S, Yusuf DS, et al (2012) Vitamin D deficiency and calcium intake in reference to increased body mass index in children and adolescents. Eur J Pediatr.

10. Calder PC, Ahluwalia N, Brouns F, Buetler T, Clement K, et al. (2011) Dietary factors and low-grade inflammation in relation to overweight and obesity. $\mathrm{Br} \mathrm{J}$ Nutr 106: S5-S78.

*Corresponding author: Demosthenes B. Panagiotakos, Associate Professor in Biostatistics-Epidemiology of Nutrition, Harokopio University of Athens, Department of Science of Dietetics-Nutrition, Athens, Greece, Tel: +30210 9549 332; Fax: +30210 9603 116; E-mail: d.b.panagiotakos@usa.net

Received March 20, 2012; Accepted March 26, 2012; Published March 29, 2012 Citation: Antonogeorgos G, Panagiotakos DB (2012) Obesity and Asthma: Is Diet a Therapeutic Mean? J Aller Ther 3:e105. doi:10.4172/2155-6121.1000e105

Copyright: (c) 2012 Antonogeorgos G, et al. This is an open-access article distributed under the terms of the Creative Commons Attribution License, which permits unrestricted use, distribution, and reproduction in any medium, provided the original author and source are credited. 
Citation: Antonogeorgos G, Panagiotakos DB (2012) Obesity and Asthma: Is Diet a Therapeutic Mean? J Aller Ther 3:e105. doi:10.4172/2155$6121.1000 \mathrm{e} 105$

Page 2 of 2

11. Kaur B, Rowe BH, Arnold E (2009) Vitamin C supplementation for asthma. Cochrane Database Syst Rev: CD000993

12. Schubert R, Kitz R, Beermann C, Rose MA, Lieb A, et al. (2009) Effect of n-3 polyunsaturated fatty acids in asthma after low-dose allergen challenge. Int Arch Allergy Immunol 148: 321-329.

13. Devereux G, Macdonald H, Hawrylowicz C (2009) Vitamin D and asthma: time for intervention? Am J Respir Crit Care Med 179: 739-740.

14. Keast DR, O'Neil CE, Jones JM (2011) Dried fruit consumption is associated with improved diet quality and reduced obesity in US adults: National Health and Nutrition Examination Survey, 1999-2004. Nutr Res 31: 460-467.

15. Arvaniti F, Priftis KN, Papadimitriou A, Papadopoulos M, Roma E, et al. (2011) Adherence to the Mediterranean type of diet is associated with lower prevalence of asthma symptoms, among 10-12 years old children: the PANACEA study. Pediatr Allergy Immunol 22: 283-289.

16. Trichopoulou A, Lagiou P (1997) Healthy traditional Mediterranean diet: an expression of culture, history, and lifestyle. Nutr Rev 55: 383-389. 